\title{
Incidence of pathogenic, likely pathogenic, and uncertain ALS variants in a clinic cohort
}

Jennifer Roggenbuck, MS, CGC, Marilly Palettas, MPH, Leah Vicini, BS, Radha Patel, BS, Adam Quick, MD, and Stephen J. Kolb, MD, PhD

Neurol Genet 2020;6:e390. doi:10.1212/NXG.0000000000000390

\section{Abstract}

\section{Objective}

To determine the incidence of amyotrophic lateral sclerosis (ALS) genetic variants in a clinicbased population.

\section{Methods}

A prospective cohort of patients with definite or probable ALS was offered genetic testing using a testing algorithm based on family history and age at onset.

\section{Results}

The incidence of pathogenic (P) or likely pathogenic (LP) variants was $56.0 \%$ in familial ALS (fALS); $11.8 \%$ in patients with ALS with a family history of dementia, and $6.8 \%$ in sporadic ALS $(p<0.001)$. C9orf72 expansions accounted for the majority $(79 \%)$ of P or LP variants in fALS cases. Variants of uncertain significance were identified in $20.0 \%$ of fALS cases overall and in $35.7 \%$ of $C 9$ orf72-negative cases. P or LP variants were detected in $18.5 \%$ of early-onset cases (onset age $<50$ years); the incidence of P or LP variants was not significantly different between family history types in this group.

\section{Conclusions}

Our data suggest that the incidence of $\mathrm{P}$ and LP variants in genes other than C9orf72 is lower than expected in Midwestern fALS cases compared with research cohorts and highlights the challenge of variant interpretation in ALS. An accurate understanding of the incidence of pathogenic variants in clinic-based ALS populations is necessary to prioritize targets for therapeutic intervention and inform clinical trial design.
Correspondence

Ms. Roggenbuck

jennifer.roggenbuck@osumc.edu

From the Department of Internal Medicine (J.R.) and Department of Neurology (J.R., A.Q., S.J.K.), The Ohio State University Wexner Medical Center; Department of Biomedical Informatics (M.P.), Center for Biostatistics, The Ohio State University Wexner Medical Center; College of Medicine (L.V., R.P.), The Ohio State University Wexner Medical Center; and Department of Biological Chemistry \& Pharmacology (S.J.K.), The Ohio State University Wexner Medical Center, Columbus. 


\section{Glossary}

ACMG = American College of Medical Genetics; ALS = amyotrophic lateral sclerosis; dALS = patients with ALS with a family history of dementia; fALS = familial ALS; LP = likely pathogenic; $\mathbf{P}=$ pathogenic; sALS = sporadic ALS; VUS = variant of uncertain significance.

Remarkable and rapid progress in the discovery of amyotrophic lateral sclerosis (ALS)-associated genes, and a growing appreciation of the genetic component of clinically sporadic ALS (sALS), has opened the door to an era of personalized, gene-targeted therapies for people with ALS. As gene-targeted therapies move through the preclinical and clinical trial pipeline, there is a pressing need to improve the practice of genetic testing and to determine the incidence of genetic forms of ALS in clinic-based populations. Despite the progress in ALS gene discovery, the offer of testing to people with ALS is not yet "standard of care," and the incidence of clinically meaningful genetic variants in clinic populations has not been studied.

A genetic etiology is reported in $\sim 70 \%$ of familial ALS (fALS) and $\sim 15 \%$ of sALS in North American research cohorts. ${ }^{1}$ However, US guidelines for ALS management do not address the offer of genetic testing, ${ }^{2}$ and European guidelines direct that testing should be offered only to patients with fALS or the SOD1 D90A phenotype. ${ }^{3}$ Lack of guidance with respect to genetic testing practices, ${ }^{4}$ in addition to challenges with testing methods and result interpretation, ${ }^{5,6}$ has likely limited the application of genetic testing in the clinic.

In this study, we determined the incidence of pathogenic (P) and likely pathogenic (LP) variants in a prospective clinicbased ALS cohort at an academic medical center, using a testing algorithm based on family history and age at onset. Second, we compared the incidence of P or LP variants in fALS, patients with ALS with a family history of dementia (dALS), sALS (having no known family history of ALS or dementia), and in early-onset cases (onset $<50$ years of age).

\section{Methods}

Patients with a diagnosis of definite or probable ALS per ElEscorial criteria were offered ALS genetic testing using a testing algorithm based on family history and age at onset (figure) over a 4-year period. A 3-generation pedigree was recorded for each patient. No attempt was made to document reported family history information via review of medical records of affected relatives. Patients who provided limited or incomplete family histories were classified as sALS, unless a family history of ALS or dementia was reported.

All patients who accepted testing $(\mathrm{n}=182)$ underwent C9orf72 repeat expansion testing as a first step. A validated assay consisting of amplicon-length analysis and repeat-primed PCR was used to detect the presence or absence of a pathogenic GGGGCC hexanucleotide repeat expansion ( $>30$ repeats). ${ }^{7}$ The amplicon-length assay was previously described. ${ }^{8}$ Two repeat-primed PCR assays, $3^{\prime}$ and $5^{\prime}$ from the hexanucleotide repeat region, were used as previously described.'

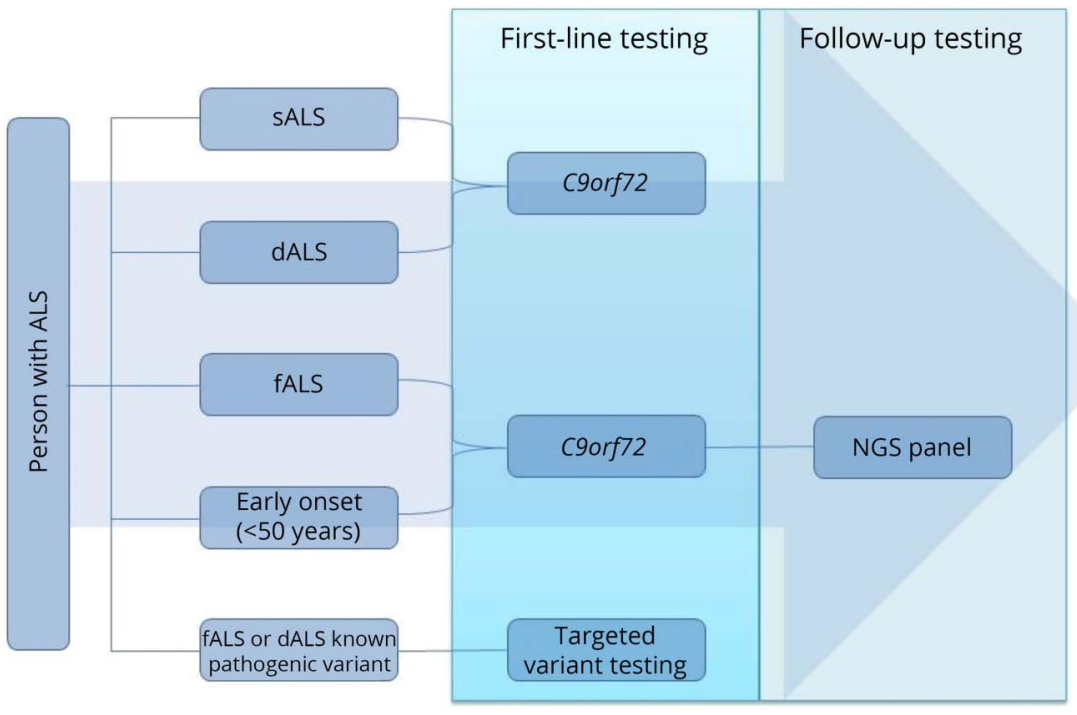

SALS, sporadic ALS with no family history of ALS or dementia; dALS, ALS with a family history of dementia but no ALS; fALS, ALS with a family history of ALS; early onset, onset of ALS symptoms $<50$ years of age. ALS $=$ amyotrophic lateral sclerosis; NGS = next generation sequencing. 
Patients with fALS and/or onset of symptoms before age 50 years, who tested negative for $C 9$ orf 72 , were offered multigene panel testing as a second step. Multigene panel testing included, at minimum, sequencing of 13 genes (ALS2, CHMP2B, DCTN1, FUS, OPTN, PFN1, SETX, SIGMAR1, SOD1, TARDBP, UBQLN2, VAPB, and VCP). Additional genes were tested in most patients (including TBK1 in 20/33, 60.6\%), reflecting gene discovery and validation during the 4-year study period (see table e-1, links.lww.com/NXG/A212 for detailed panel data). Sequencing was performed using Illumina technology. For cases tested during the first year of the study, $96 \%$ of targeted regions were covered at a depth of 20x. During the remainder of the study period, all targeted regions were sequenced with $\geq 50 \times$ depth or supplemented with additional analysis. Reads were aligned to a reference sequence (GRCh37), and sequence changes were identified and interpreted in the context of a single clinically relevant transcript. Enrichment and analysis focused on the coding sequence and 10bp of flanking intronic sequence. All clinically significant observations were confirmed by orthogonal technologies, including Sanger sequencing, Pacific Biosciences Single Molecule, Real-time sequencing, Multiplex Ligation-dependent Probe Amplification, Multiplex Ligation-dependent Probe Amplification-seq, and Array Comparitive Genomic Hybridization.

Descriptive statistics were used to summarize demographic and clinical characteristics, as well as incidence of $\mathrm{P}$ and LP variants, overall and by family history classification (fALS, dALS, or sALS). Comparisons between groups were made using a $\chi^{2}$ or Fisher exact test, where appropriate. Analyses were performed in SAS 9.4 (SAS Institute, Cary, NC).

\section{Data availability}

Study data are available on request.

\section{Standard protocol approvals, registrations,} and patient consents

The Office of Responsible Research Practices at The Ohio State University Medical Center considers this project exempt from review.

\section{Results}

Of 167 persons with ALS who completed the testing algorithm, the majority reported Caucasian ancestry (93\%), followed by African American (3\%), and Asian (1\%); 4\% reported other or mixed ancestry. The median age at onset of motor neuron disease symptoms was 61 years (range $20-83$ years); $16.2 \%$ were classified as early onset with onset $<50$ years. Fifteen percent $(25 / 167)$ were classified as fALS (having a positive family history of ALS in a 1st-,

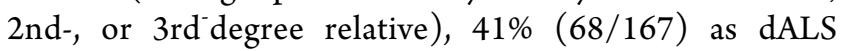
(having a positive family history of dementia of any type in a 1 st- or 2 nd-degree relative), and $44.0 \%(74 / 167)$ as sALS (table 1).

Variants classified as P or LP were identified in $56.0 \%$ of fALS cases; the majority being C9orf72 expansions (11/25, 44.0\%), followed by P or LP variants in SOD1 (2/25, 8.0\%), and FUS $(1 / 25,4.0 \%)$. Variants of uncertain significance (VUS) were identified in $20.0 \%$ of fALS cases overall and in $35.7 \%$ of C9orf72-negative cases. C9orf72 expansions were detected in 8/68 (11.8\%) dALS cases. Among sALS cases, P or LP variants were identified in 5/74 (6.8\%), including 3 in C9orf 72 (4.0\%), 1 in SOD1 (1.4\%), and 1 in FUS (1.4\%). The overall incidence of $\mathrm{P}$ or LP variants was significantly different among fALS, dALS, and sALS cases $(56.0 \%, 11.8 \%$, and $6.8 \%$, respectively; $p<0.001$ ) (table 2).

Table 1 Demographic and disease characteristics of the tested cohort

\begin{tabular}{|c|c|c|c|c|}
\hline Characteristic & fALS $(n=25)$ & dALS $(n=68)$ & sALS $(n=74)$ & Total $(n=167)$ \\
\hline \multicolumn{5}{|l|}{ Ethnicity } \\
\hline African & $1(4 \%)$ & $2(3 \%)$ & $2(3 \%)$ & $5(3 \%)$ \\
\hline Asian/Native American & $0(0 \%)$ & $0(0 \%)$ & $1(1 \%)$ & $1(1 \%)$ \\
\hline European & $24(96 \%)$ & $64(94 \%)$ & $67(91 \%)$ & 155 (93\%) \\
\hline Mixed/unknown/other & $0(0 \%)$ & $2(3 \%)$ & $4(5 \%)$ & $6(4 \%)$ \\
\hline \multicolumn{5}{|l|}{ Age at onset } \\
\hline Median [IQR] (min, max) & $60[50,61](34,70)$ & $63[54,67](20,80)$ & $61.5[52,70](21,83)$ & $61[52,69](20,83)$ \\
\hline \multicolumn{5}{|l|}{ Age at testing } \\
\hline Median [IQR] (min, max) & $61[53,64](37,71)$ & $65[56,70](24,80)$ & $65[55,71](31,84)$ & $64[55,70](24,84)$ \\
\hline \multicolumn{5}{|l|}{ Onset $<50 y$} \\
\hline No & $19(76 \%)$ & $59(87 \%)$ & $62(84 \%)$ & $140(84 \%)$ \\
\hline Yes & $6(24 \%)$ & $9(13 \%)$ & $12(16 \%)$ & $27(16 \%)$ \\
\hline
\end{tabular}

Abbreviations: $\mathrm{dALS}=$ patients with ALS with a family history of dementia; fALS = familial ALS; IQR = interquartile range; $\mathrm{SALS}=$ sporadic ALS. 
Table 2 Summary outcomes of the ALS genetic testing algorithm

\begin{tabular}{|c|c|c|c|c|c|}
\hline ALS family history & Test type & Positive result & Negative result & VUS/intermediate result & Positive (\%) \\
\hline \multirow[t]{2}{*}{ fALS $(n=25)$} & C9 & 11 & 0 & 0 & $14(56.0 \%)$ \\
\hline & C9 + panel & 3 & 6 & 5 & \\
\hline \multirow[t]{2}{*}{ dALS $(n=68)$} & C9 & 8 & 52 & 0 & $8(11.8 \%)$ \\
\hline & C9 + panel & 0 & 6 & 2 & \\
\hline \multirow[t]{2}{*}{ sALS $(n=74)$} & C9 & 3 & 59 & 1 & $5(6.8 \%)$ \\
\hline & C9 + panel & 2 & 9 & 0 & \\
\hline \multirow[t]{2}{*}{ Total $(n=167)$} & C9 & 22 & 111 & 1 & $27(16.2 \%)$ \\
\hline & C9 + panel & 5 & 21 & 7 & \\
\hline
\end{tabular}

Abbreviations: ALS = amyotrophic lateral sclerosis; $d A L S=$ patients with ALS with a family history of dementia; fALS = familial ALS; sALS = sporadic ALS; VUS = variant of uncertain significance.

The subgroup of early-onset cases was examined separately; 6 (22.2\%) were classified as fALS, 9 (33.3\%) as dALS, and 12 (44.4\%) as sALS. P or LP variants were detected in $18.5 \%$ of early-onset cases, with an LP SOD1 variant in 1/6 (16.7\%) fALS cases, a P FUS variant in 1/9 (11.1\%) dALS cases, and an LP SOD1 variant, a P FUS variant, and C9orf 72 expansion in $3 / 12$ (25.0\%) sALS cases, respectively. The rate of P or LP variant detection was not substantially different between family history types in the early-onset group (fALS: 16.7\%, dALS: $11.1 \%$, sALS: $25 \% ; p=0.823$ ) (table 3 ).

\section{Discussion}

Test outcome data from our clinic population suggest that the incidence of $\mathrm{P}$ or LP variants in Midwestern fALS cases may be lower than that reported in research cohorts. In particular, the lower incidence of pathogenic variants in SOD1 and genes other than C9orf72 is notable. Possible explanations for this observation include the following: (1) the standards used to guide the interpretation of genetic variants identified in research may differ from those applied in clinical testing; (2) research cohorts may be enriched for fALS cases from "high penetrance" families; and (3) the incidence of pathogenic variants in specific genes varies by geographic ancestry. In favor of the first possibility, VUS were identified in $35.7 \%$ of our C9orf72-negative fALS cases; such variants may be considered causative in research testing but may not reach the American College of Medical Genetics (ACMG) evidentiary standards for LP or P classification in clinical testing. ACMG criteria for variant pathogenicity rely on several lines of published evidence, including wellestablished functional studies demonstrating a deleterious effect, and cosegregation with disease in multiple affected individuals. ${ }^{10}$ Functional data are not available for many ALS genes, and segregation data are limited because affected relatives are not often available for testing. Efforts are underway to revise ACMG criteria for ALS variant interpretation using disease-specific and gene-specific data. ${ }^{11}$

Table 3 Outcomes of the ALS genetic testing algorithm for individuals with early onset $(<50 \mathrm{y})$

\begin{tabular}{llllll}
\hline ALS family history & Test type & Positive result & Negative result & VUS/intermediate result & Positive (\%) \\
\hline fALS $(\mathbf{n}=\mathbf{6})$ & C9 & 0 & 0 & 0 & $1(16.7 \%)$ \\
\hline dALS $(\mathbf{n}=\mathbf{9})$ & C9 + panel & 1 & 3 & 0 & $1(11.1 \%)$ \\
\hline & C9 & 1 & 0 & 2 & 0 \\
\hline SALS $(\mathbf{n}=\mathbf{1 2})$ & C9 + panel & 0 & 6 & 0 & $3(25.0 \%)$ \\
\hline & C9 & 1 & 0 & 0 \\
\hline Total $(\mathbf{n}=\mathbf{2 7})$ & C9 + panel & 2 & 9 & 4 \\
\hline
\end{tabular}

Abbreviations: ALS = amyotrophic lateral sclerosis; $\mathrm{dALS}=$ patients with ALS with a family history of dementia; fALS = familial ALS; $S A L S=$ sporadic $A L S ;$ VUS = variant of uncertain significance. 
We believe that our initial test outcome data generally support the use of the genetic testing algorithm shown in the figure. This approach includes the offer of C9orf 72 testing to all patients. As noted above, current US care guidelines do not address genetic testing, and European recommendations specify that genetic testing be offered only to patients with a family history of ALS or the SOD1 D90A phenotype. However, our data suggest that limiting the offer of C9orf 72 testing to patients with a positive family history of ALS would lead to approximately half of $C 9$ orf 72 carriers being missed. In our data set, 11/22 (50.0\%) C9orf72-positive cases had a family history of ALS. Similarly, in a published clinic-based research cohort, $60 \%$ of C9orf72-positive cases had a family history of ALS. ${ }^{12}$ We believe that the offer of C9orf72 to all patients with ALS is therefore justified, particularly given the availability of $\mathrm{C} 9$ orf 72 targeted therapeutic trials and relatively low cost of testing.

A potential limitation of the algorithm is that it would miss cases that are positive for the C9orf72 repeat expansion in addition to a second pathogenic variant, which has been reported. ${ }^{12}$ More data are needed regarding potential oligogenic inheritance in ALS, which may affect the approach to testing. In the meantime, clinicians may wish to consider both C9orf72 repeat expansion testing in addition to multigene panel testing in fALS cases and should certainly do so if there is a family history of ALS in more than 1 branch of the family. Finally, emerging data suggest that ATXN2 expansions (known to cause spinocerebellar ataxia type 2) also cause ALS and may be as common in ALS cohorts as pathogenic variants in TARDBP. ${ }^{13}$ Therefore, clinicians should consider adding this assay for fALS and early-onset cases in whom no genetic etiology is found after C9orf 72 repeat expansion and multigene panel testing.

We propose the designation of dALS to denote ALS patients whose family history is negative for ALS but positive for dementia. Although FTD is the only type of dementia known to share a genetic etiology with ALS, the specific dementia type reported in family members cannot be reliably determined from family history information, or even necessarily from review of medical records. We believe that our data warrant this inclusive classification, given the higher rate of C9orf72 expansions identified in this group (11.8\%) compared with sporadic cases with no family history of ALS or dementia (6.8\%). The importance of a positive family history of any dementia in identifying potential cases of C9orf72 in clinically sALS was shown in another clinic cohort study in which $60 \%$ of sALS cases who tested positive for C9orf72 had a positive family history of dementia. Finally, our data support the offer of testing to patients with early onset of ALS symptoms, irrespective of family history, given that the positive yield of testing in this group (18.5\%) was second only to fALS (56.0\%) among the clinical categories examined. These data, if replicated, could also be useful in counseling patients with ALS who are considering genetic testing.

The majority of individuals in this study reported European ancestry, which limits the applicability of our data to other populations. The variable ethnogeographic incidence of specific pathogenic variants (e.g., the C9orf72 expansion) ${ }^{14}$ may warrant consideration of population-specific testing approaches. Our current understanding of the familial clustering and genetic basis of ALS is primarily derived from the study of Caucasian individuals and reflects a significant disparity in ALS research and care. Further study is needed regarding the genetic basis of ALS in ethnically and geographically diverse populations. An accurate understanding of the incidence of clinically meaningful variants in different ALS populations is necessary to prioritize targets for therapeutic intervention and inform clinical trial design.

We advocate the offer of $C 9$ orf 72 testing to all persons with ALS and multigene testing as a second step for those with fALS or onset of symptoms before age 50 years. However, the incidence of $\mathrm{P}$ or LP variants in genes other than C9orf72 (including SOD1) may be lower than expected from published research cohorts, and many cases of fALS may remain unsolved with current testing and interpretation standards. Consistent, equitable genetic testing practices, and an accurate understanding of the incidence of clinically meaningful variants in clinic-based, geographically diverse ALS populations, are necessary as the community of ALS patients and clinicians prepares for the clinical trials and approved therapies of the future.

\section{Study funding}

This project has been supported in part by the Neuroscience Research Institute at The Ohio State University.

\section{Disclosure}

Disclosures available: Neurology.org/NG.

\section{Publication history}

Received by Neurology: Genetics July 16, 2019. Accepted in final form December 2, 2019.

\begin{tabular}{|c|c|c|c|}
\hline Name & Location & Role & Contribution \\
\hline $\begin{array}{l}\text { Jennifer } \\
\text { Roggenbuck, } \\
\text { MS, CGC }\end{array}$ & $\begin{array}{l}\text { The Ohio State } \\
\text { University } \\
\text { Wexner Medical } \\
\text { Center }\end{array}$ & Author & $\begin{array}{l}\text { Designed and } \\
\text { conceptualized the study; } \\
\text { data collection and } \\
\text { analysis; and drafted the } \\
\text { manuscript for } \\
\text { intellectual content }\end{array}$ \\
\hline $\begin{array}{l}\text { Marilly } \\
\text { Palettas, } \\
\text { MPH }\end{array}$ & $\begin{array}{l}\text { The Ohio State } \\
\text { University } \\
\text { Wexner Medical } \\
\text { Center }\end{array}$ & Author & $\begin{array}{l}\text { Data collection and } \\
\text { analysis }\end{array}$ \\
\hline $\begin{array}{l}\text { Leah Vicini, } \\
\text { BS }\end{array}$ & $\begin{array}{l}\text { The Ohio State } \\
\text { University } \\
\text { Wexner Medical } \\
\text { Center }\end{array}$ & Author & Data collection \\
\hline $\begin{array}{l}\text { Radha Patel, } \\
\text { BS }\end{array}$ & $\begin{array}{l}\text { The Ohio State } \\
\text { University } \\
\text { Wexner Medical } \\
\text { Center }\end{array}$ & Author & Data collection \\
\hline
\end{tabular}


Appendix (continued)

\begin{tabular}{llll}
\hline Name & Location & Role & Contribution \\
\hline $\begin{array}{l}\text { Adam Quick, } \\
\text { MD }\end{array}$ & $\begin{array}{l}\text { The Ohio State } \\
\text { University } \\
\text { Wexner Medical } \\
\text { Center }\end{array}$ & Author & $\begin{array}{l}\text { Drafting and revision for } \\
\text { intellectual content }\end{array}$ \\
& $\begin{array}{l}\text { The Ohio State } \\
\text { Stephen J. } \\
\begin{array}{l}\text { Kolb, MD, } \\
\text { PhD }\end{array}\end{array}$ & Author & \\
& $\begin{array}{l}\text { Wexner Medical } \\
\text { Center }\end{array}$ & & $\begin{array}{l}\text { Drafting and revision for } \\
\text { intellectual content }\end{array}$ \\
& & & \\
\hline
\end{tabular}

\section{References}

1. Chia R, Chiò A, Traynor BJ. Novel genes associated with amyotrophic lateral sclerosis: diagnostic and clinical implications. Lancet Neurol 2018;17:94-102.

2. Miller RG, Jackson CE, Kasarkis EJ, et al. Practice parameter update: the care of the patient with amyotrophic lateral sclerosis: multidisciplinary care, symptom management, and cognitive/behavioral impairment (an evidence-based review): report of the Quality Standards Subcommittee of the American Academy of Neurology. Neurology 2009;73:1227-1233.

3. Andersen PM, Abrahams S, Borasio GD, et al. EFNS guidelines on the clinical management of amyotrophic lateral sclerosis (MALS)-revised report of an EFNS task force. EFNS Task Force on Diagnosis and Management of Amyotrophic Lateral Sclerosis. Eur J Neurol 2012;19:360-375.
4. Klepek H, Nagaraja H, Goutman SA, Quick A, Kolb SJ. Lack of consensus in ALS genetic testing practices and divergent views between ALS clinicians and patients. Amyotroph Lateral Scler Frontotemporal Degener 2019;20:216-221.

5. Klepek H, Goutman SA, Quick A, Kolb SJ, Roggenbuck J. Variable reporting of C9orf72 and a high rate of uncertain results in ALS genetic testing. Neurol Genet 2019;5:e301.

6. Crook A, McEwen A, Fifita JA, et al. The C9orf72 hexanucleotide repeat expansion presents a challenge for testing laboratories and genetic counseling. Amyotroph Lateral Scler Frontotemporal Degener 2019;20:310-316.

7. Akimoto C, Volk AE, Blitterswijk MV, et al. A blinded international study on the reliability of genetic testing for GGGGCC-repeat expansions inC9orf72reveals marked differences in results among 14 laboratories. J Med Genet 2014;51:419-424.

8. Dejesus-Hernandez M, Mackenzie IR, Boeve BF, et al. Expanded GGGGCC hexanucleotide repeat in noncoding region of C9ORF72 causes chromosome 9p-linked FTD and ALS. Neuron 2011;72:245-256.

9. Cleary EM, Pal S, Azam T, et al. Improved PCR based methods for detecting C9orf72 hexanucleotide repeat expansions. Mol Cell Probes 2016;30:218-224.

10. Richards S, Nazneen A, Bale S, et al. Standards and guidelines for the interpretation of sequence variants: a joint consensus recommendation of the American College of Medical Genetics and Genomics and the Association for Molecular Pathology. Genet Med 2015; 17:405-423.

11. Leighton D, de Araujo Martins Moreno C, Lehrer H, et al. Assessing ACMG criteria for the classification of reported ALS gene mutations: utility, pitfalls and recommendations. Neurology 2019;92(15 suppl).

12. Umoh ME, Fournier $\mathrm{C}, \mathrm{Li} \mathrm{Y}$, et al. Comparative analysis of C9orf72 and sporadic disease in an ALS clinic population. Neurology 2016;87:1024-1030.

13. Moreno C, Hoover B, Likanje M, et al. Pathogenic ATXN2 repeat expansions are as common as TARDBP mutations in large ALS cohorts. In: Presented at Northeast ALS Consortium Conference. Clearwater Beach, FL; 2019.

14. Pliner H, Mann D, Traynor BJ. Searching for Grendel: origin and global spread of the C9ORF72 repeat expansion. Acta Neuropathol 2014;127:391-396. 